DOI: 10.1136/annrheumdis-2018-eular.5407

\section{OP0181 PATIENT PERSPECTIVES OF PEOPLE WITH PRIMARY SJÖGREN'S SYNDROME: A MULTICENTRE QUALITATIVE EUROPEAN STUDY}

J. Unger ${ }^{1}$, M. Mattsson ${ }^{2,3}$, R. Dragoi ${ }^{4}$, C. Boström ${ }^{3}$, F. Buttgereit ${ }^{5}$, A. Lackner ${ }^{6}$, T. Witte ${ }^{7}$, B. Raffeiner ${ }^{8}$, P. Peichl ${ }^{9}$, J. Hermann ${ }^{6}$, C. Dejaco ${ }^{6,10}$, T. Stamm $^{11} .{ }^{1} F H$ JOANNEUM, University of Applied Sciences, Bad Gleichenberg, Austria;

${ }^{2}$ Sunderby Hospital, Department of Physiotherapy, Luleå; ${ }^{3}$ Karolinska Institutet, Department of Neurobiology, Care Sciences and Society, Stockholm, Sweden; ${ }^{4}$ Victor Babes University of Medicine and Pharmacy Timisoara, Timisoara, Romania; ${ }^{5}$ Charitè University Medicine, Berlin, Germany; ${ }^{6}$ Medical University Graz, Graz, Austria; ${ }^{7}$ Medical University of Hannover, Hannover, Germany; ${ }^{8}$ Hospital of Bolzano, Bolzano, Italy; ${ }^{9}$ Evangelic Hospital of Vienna, Vienna, Austria; ${ }^{10}$ Hospital of Bruneck, Bruneck, Italy, ${ }^{11}$ Medical University Vienna, Vienna, Austria

Background: Primary Sjögren's syndrome (pSS) is one of the most common systemic autoimmune disorders and leads to an impaired health related quality of life. However, treatment mainly focuses on the management of physical manifestations. Little is known about the lived experiences of people with pSS, including the impact on people's life, functioning and their social relationships.

Objectives: To explore the perspectives of people with pSS from different European countries with various cultural backgrounds in order to achieve a broad understanding of concepts that are important and meaningful to people with pSS. This study is a part of a project which aims to evaluate the coverage of the patient perspectives by patient reported outcome measures in pSS, which is funded by the Austrian Association of Rheumatology.

Methods: A multicentre focus group study was performed in five European countries, namely Austria, Germany, Italy, Romania and Sweden. Patients were recruited from the outpatient clinics of the local centres. Focus groups were chaired by a trained moderator and followed an interview guide which included questions about impairments and limitations in body structures, body functions, activities and participation as well as contextual factors and resources, such as coping strategies. Focus groups were audiotaped and transcribed. We conducted a content-analysis of each focus group and subsequently combined the extracted concepts from each country, using the International Classification of Functioning, Disability and Health as a frame of reference.

Results: A total of 12 focus groups was conducted in seven participating centres in five countries. Fifty people (48 women; $96 \%$ ) with pSS participated in the focus groups (ranging from two to four groups per country). All focus groups had a total duration of $1030 \mathrm{~min}$ and resulted in 252 pages of transcript. From qualitative analysis we derived concepts meaningful to people with pSS from all countries, especially those concepts that were linked to a physical dimension. However, we identified differences in the description of these experiences in daily life, for example for pain-concerning sensations or for the impact on social relationships. Furthermore, the attitudes towards the treatment and towards the disease differed between the participants. People with pSS had various coping strategies, such as gaining more knowledge about the disease or utilising non-pharmacological treatment.

Conclusions: This is the first multicentre qualitative European study which investigated the patient perspectives in pSS with a cross-cultural understanding. Clinicans, health professionals and researchers need to know about the perspectives, experiences and needs of people with pSS in order to ensure a comprehensive treatment.

Disclosure of Interest: None declared

DOI: 10.1136/annrheumdis-2018-eular.4699

THURSDAY, 14 JUNE 2018

\section{The crystal maze - etiology and management}

\section{OP0182 GOUT AND DEMENTIA IN THE ELDERLY: A MEDICARE CLAIMS STUDY}

\section{J.A. Singh, J.D. Cleveland. University of Alabama at Birmingham, Birmingham} USA

Background: The pursuit of a link between gout/hyperuricemia and dementia has led to contradictory results. Most observational studies, including populationbased studies, showed that hyperuricemia was associated with a higher risk of dementia and less cognitive dysfunction, while a few studies found hyperuricemia to be associated with a lower risk of dementia. Recently, a large French population-based study in the elderly (65 years or older) showed that hyperuricemia was associated with a higher risk of dementia and with MRI changes of ageing in the brain.
Objectives: To assess whether gout in the elderly is associated with a risk of incident dementia.

Methods: We used the $5 \%$ Medicare claims data for this observational cohort study. We used multivariable-adjusted Cox proportional hazard models to assess the association of gout with incident dementia, adjusting for potential confounders/covariates including demographics (age, race, gender), comorbidities (Charlson-Romano comorbidity index), and medications commonly used for cardiac diseases (statins, beta-blockers, diuretics, and angiotensin converting enzyme (ACE)-inhibitors) and gout (allopurinol and febuxostat).

Results: In our cohort of 1.23 million Medicare beneficiaries, 65324 had incident dementia. The crude incidence rates in people without and with gout were 7.36 and 13.58 per 1000 person-years, respectively. In multivariable-adjusted analyses, gout was independently associated with a significantly higher hazard ratio of incident dementia, with a hazard ratio [HR] of $1.17(95 \% \mathrm{Cl}, 1.13,1.21)$; sensitivity analyses confirmed the main findings. Compared to age 65 to $<75$ years, older age groups were associated with 3.3 and 6.3-fold higher hazards of dementia; hazards were also higher for females, Black race or people with higher medical comorbidity.

Subgroup analyses indicated that gout was significantly associated with dementia in patients without key comorbidities (CAD, hyperlipidemia, CVD, diabetes, hypertension) with HR ranging 1.20-1.57, but not in patients with each of these comorbidities, except CAD, with HR 0.97-1.07 (table 1).

Abstract OP0182 - Table 1. Association of gout with incident dementia, in pre-defined subgroups of presence/absence of CAD, hyperlipidemia, CVD, diabetes or hypertension

\begin{tabular}{|c|c|c|c|c|}
\hline & \multicolumn{2}{|c|}{$\begin{array}{l}\text { Multivariable-adjusted } \\
\text { (Model 1) }\end{array}$} & \multicolumn{2}{|c|}{$\begin{array}{l}\text { Multivariable-adjusted } \\
\text { (Model 1) }\end{array}$} \\
\hline & $\mathrm{HR}(95 \% \mathrm{Cl})$ & P-value & $\mathrm{HR}(95 \% \mathrm{Cl})$ & $\begin{array}{c}\mathrm{P}- \\
\text { value }\end{array}$ \\
\hline & No CAD & & CAD & \\
\hline \multirow[t]{2}{*}{ Gout } & $1.20(1.15-1.25)$ & $<0.0001$ & $1.07(1.01-1.14)$ & 0.032 \\
\hline & $\begin{array}{c}\text { No } \\
\text { Hyperlipidemia }\end{array}$ & & Hyperlipidemia & \\
\hline \multirow[t]{2}{*}{ Gout } & $1.33(1.27-1.40)$ & $<0.0001$ & $1.02(0.97,1.07)$ & 0.56 \\
\hline & No CVD & & CVD & \\
\hline \multirow[t]{2}{*}{ Gout } & $1.23(1.18-1.28)$ & $<0.0001$ & $0.97(0.90-1.05)$ & 0.46 \\
\hline & No Diabetes & & Diabetes & \\
\hline \multirow[t]{2}{*}{ Gout } & $1.24(1.19-1.29)$ & $<0.0001$ & $1.04(0.98-1.10)$ & 0.23 \\
\hline & No Hypertension & & Hypertension & \\
\hline Gout & $1.57(1.46-1.68)$ & $<0.0001$ & $1.01(0.97-1.05)$ & 0.76 \\
\hline
\end{tabular}

Gout ${ }^{\star}$ CAD p-value $<0.0001$; Gout ${ }^{\star}$ hyperlipidemia p-value $<0.0001$; Gout ${ }^{\star}$ CVD $p$ value $<0.0001$;

Gout ${ }^{*}$ diabetes p-value $<0.0001$; Gout ${ }^{\star}$ hypertension p-value $<0.0001$;

Conclusions: Gout was independently associated with $17 \%-20 \%$ higher risk of incident dementia in the elderly. Future studies need to understand the pathogenic pathways involved in this increased risk.

Acknowledgements: This material is the result of work supported by research funds from the Division of Rheumatology at the University of Alabama at Birming ham and the resources and use of facilities at the Birmingham VA Medical Centre, Birmingham, Alabama, USA.

Disclosure of Interest: J. Singh Grant/research support from: Takeda, Savient Consultant for: Savient, Takeda, Regeneron, Merz, Iroko, Bioiberica, Crealta/ Horizon and Allergan pharmaceuticals, WebMD, UBM LLC and the American College of Rheumatology, J. Cleveland: None declared

DOI: 10.1136/annrheumdis-2018-eular.7595

\section{OP0183 ASSOCIATION BETWEEN HFE GENOTYPES AND CLINICAL SEVERITY CHARACTERISTICS OF CALCIUM PYROPHOSPHATE CRYSTAL ARTHRITIS}

F. Perez-Ruiz, S.P. Chinchilla, J. Atxotegi. Rheumatology Division of Cruces Hospital and University of the Basque Country, Baracaldo, Spain

Background: Several metabolic disturbances that reduce the activity of pyrophosphatases have been associated with development of calcium pyrophosphate crystal arthritis (CPPCA), but there is scarce data on their influence on clinical manifestations, as such disease-specific variables are not recorded in most available databases.

Objectives: To evaluate factors associated to severity of clinical joint involvement in patients with definite CPPCA

Methods: Transversal study with prospective recruitment of cases (patients with CPPCA diagnosis confirmed by microscopy showing CPP crystals within 